\title{
Interference alignment algorithm in coordinated multipoint transmission system
}

\author{
Yun Liu \\ College of Information Science and Technology, Qingdao University of Science and Tech nology, QUST \\ Qingdao,266000, China \\ E-mail: Iyun-1027@163.com

\section{Hui Qiao'} \\ College of Information Science and Technology, Qingdao University of Science and Tech nology, QUST \\ Qingdao, 266000, China \\ E-mail:qiao8612080163.com
}

Benefit by using orthogonal frequency division multiplexing (OFDM), LTE-Advanced system can eliminate intra-cell interference, however it still cannot decrease inter-cell interference (ICI). As a realization of CoMP, interference alignment (IA) attracts wide attention recently, which can obtain the optimal transmitting degree of freedom in multi-cell systems. On the basis of CoMPMU-MIMO system to LTE-Advanced which uses BBU+RRU, a novel optimized MSV-TZ precoding scheme is proposed for the downlink interference channel of cellular MIMO system. MSV-TZ pre-coding scheme is proposed under the premise of eliminating the interference of other cells of the residential customers. Not only concerned the performance of SU-MIMO, but also focus to improve the performance of MU-MIMO, this precoding matrixes are designed by selecting the precoding vectors which are closest to the maximal Eigen-channels, which improves the channel condition of the desired signal and strengthens the desired signal, in order to achieve a higher system throughput. By system level performance evaluation, this new CoMP-MU-MIMO transmission scheme has the huge advantage of increasing the average cell throughput and the cell edge user throughput.

CENet2015

12-13 September 2015

Shanghai, China

${ }^{1}$ Speaker 


\section{Introduction}

LTE-Advanced focuses on the data side of the flat low latency and network, aims to provide users with high-rate services and improve spectrum efficiency, especially at the cell edge spectrum efficiency. Based on orthogonal frequency division multiplexing LTE-A system, although the user can completely eliminate intra-cell interference, the natural inter-cell interference cannot be avoided [1], especially at the cell edge user interference. Current methods of inter-cell interference varied, but truly effective and feasible is coordinated multipoint transmission technology, because of its focus on user data and shared channel status collaboration between the various base stations and the use of pre-coding technology [2], between cells interference signals into useful information that can effectively improve the spectrum utilization edge users and cell edge performance. As a promising technique, the interference alignment (IA) not only effectively reduces interference, but also raises capacity of a wireless communication network [3]. An interference alignment scheme put forwarded in this paper, is used in a network with multiple cells and multiple multiple-input and multiple-output (MIMO) users under a Gaussian interference broadcast channel (IFBC) scenario. Our focus is on the overheads in a system with quantized channel state feedback from the receiver to the transmitter and on the influence on non-ideal hardware.

\section{CoMP System Model}

Our proposal bases on CoMP multiple base stations which uses BBU+RRU (Remote Radio Unit), depicted in Figure 1. With respect to others base station cooperative mode, CoMP technology shares user data and channel state more reasonable between each cooperative base station, turns the inter-cell interference into a useful signal by the use of pre-coding technique, which is greatly improved system capacity and spectrum utilization. [4]

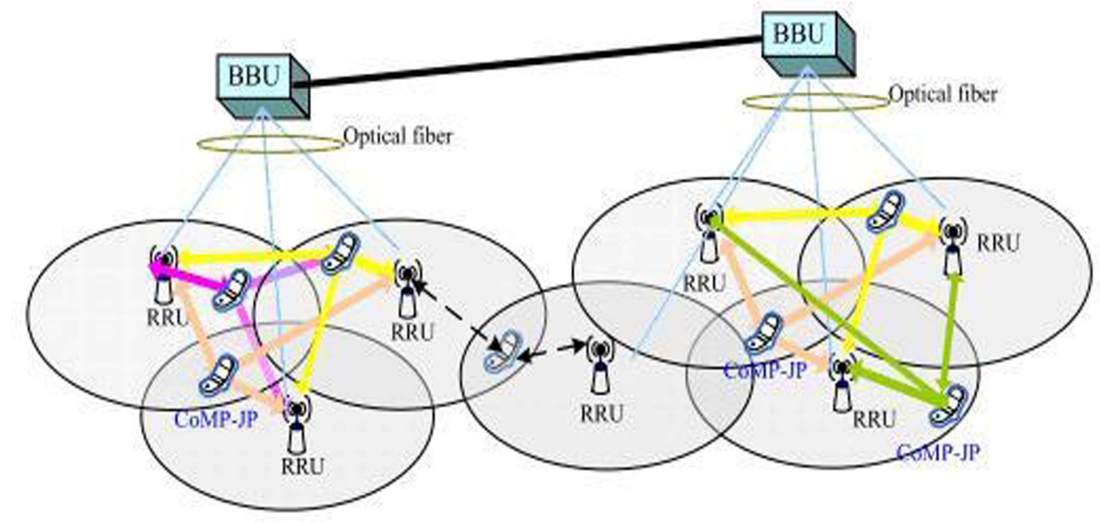

Figure 1: Network topology of BBU+RRU based CoMP system

In order to make better use of spectrum resources and improve cell-edge spectrum efficiency, the paper not only abandoned single-user joint transmission, but also uses CoMPMU-MIMO mechanism to provide joint service for multiple users, then we proposes multiuser pre-coding joint transmission improvements [5]. In this system, each base station of the radio frequency unit has pulled away $n_{t}$ transmit antennas, corresponding, $n_{r}$ represents the number of user reception antenna. Each remote radio unit can handle a physical layer cell formats. In the Figure 2, the M cooperative RRUs which compose a CoMP-RRU and these served $M$ paired UEs can form a $\left(\mathrm{Mn}_{\mathrm{r}}\right) \times\left(\mathrm{Mn}_{\mathrm{t}}\right)$ virtual MIMO system in the down link. These UE are paired and jointly scheduled in the same frequency [6]. 

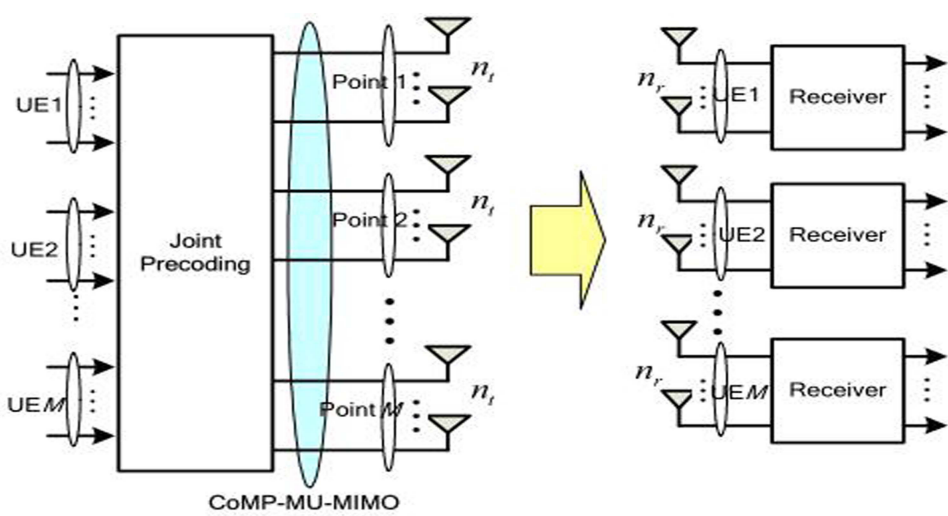

Figure 2: Downlink virtual MIMO system structure

$H_{u}[n]=I_{u}[n] F_{u}[n]$ is the channel matrix from CoMP-RRU to the $u$-th user in the UE-group on the $n$-th subcarrier. $I_{u}[n]$ denoted as the normalized complex channel gain is a $n_{r} \times\left(M_{t}\right)$ dimension matrix[7]. Below is $\mathrm{F}_{\mathrm{u}}[\mathrm{n}]$ which is a $\left(\mathrm{Mn}_{\mathrm{t}} \times \mathrm{Mn}_{\mathrm{t}}\right)$ diagonal matrix:

$$
F_{N}[\mathrm{n}]=\operatorname{diag}\left(\sqrt{P_{u, 1}[n]}, \sqrt{P_{u, 2}[n]}, \cdots \sqrt{P_{u, M n t}[n]}\right)
$$

The average received signal power for the $\mathrm{u}$-th $\mathrm{UE}$ received at the $\mathrm{i}$-th receive antenna is denoted by $\mathrm{P}_{\mathrm{u}, \mathrm{i}}[\mathrm{n}]=\operatorname{Ptx}_{\mathrm{u}}[\mathrm{n}] \times$ Pathloss $_{\mathrm{u}, \mathrm{i}} \times$ Shadowing $_{\mathrm{u}, \mathrm{i}}\left(1 \leq \mathrm{i} \leq \mathrm{Mn}_{\mathrm{t}}\right)[7]$. Accordingly, the composite channel matrix of the cooperative $\left(\mathrm{Mn}_{\mathrm{r}}\right) \times\left(\mathrm{Mn}_{\mathrm{t}}\right)$ virtual MIMO system on the $\mathrm{n}$-th subcarrier is given by [8]:

$$
H[n]=\left[\begin{array}{c}
H_{1}[n] \\
H_{2}[n] \\
\vdots \\
H_{M}[n]
\end{array}\right]
$$

The u-th UE's transmitted vector on the $\mathrm{n}$-th subcarrier is given by:

$$
D_{u}[n]=\left[D_{u, 1}[n] \quad D_{u, 2}[n] \quad \cdots \quad D_{u, l}[n]\right]^{T}
$$

Where $\mathrm{D}_{\mathrm{u}}[\mathrm{n}]$ is a pre-coding matrix of UE, and 1 represents the number of the plies. So the data sent vector on the n-th subcarrier at the CoMP is given by:

$$
T[n]=\sum_{u=1}^{M} B[n] D_{u}[n]=B[n] D[n]
$$

The received vector on the $\mathrm{u}$-th subcarrier is given by:

$$
R_{u}[n]=H_{u}[n] \sum_{i=1}^{M} B_{i}[n] D_{i}[n]+N[n]
$$

Where $N[n]$ is a $n_{r} * 1$ dimension noise vector, each element of which is a zero-mean complex additive.

\section{MSV-TZ Pre-coding Algorithm}

In order to perform the improvement of the algorithm based on CJ pre-coding algorithm, this article conduct the research to the $\mathrm{CJ}$ pre-coding algorithm, and then analyze its advantages and disadvantages. In the CoMP system which $\mathrm{M}=3$, the freedom of system transmitting degree is $3 n_{t} / 2$. At this time, in order to send $3 n_{t} / 2$ data stream independently, $B_{i}[n]$ is a $n_{t} / 2 * 1$ dimension matrix and $D_{i}[n]$ is a $n_{t} / 2 * n_{t} / 2$ dimension matrix. In order to decode $n_{t} / 2$ data stream from the received signal $B_{i}[n]$ which dimension is $n_{t} / 2$, the interference signal vector must be independent 
of other useful signals, and up to $n_{t} / 2$ dimension. Formula (3.1) is the necessary conditions of pre-coding algorithm in the system.

$$
\begin{aligned}
\operatorname{span}\left[\mathrm{H}_{12} \mathrm{~B}_{2}\right] & =\operatorname{span}\left[\mathrm{H}_{13} \mathrm{~B} 3\right] \\
\operatorname{span}\left[\mathrm{H}_{21} \mathrm{~B}_{1}\right] & =\operatorname{span}\left[\mathrm{H}_{23} \mathrm{~B}_{3}\right] \\
\operatorname{span}\left[\mathrm{H}_{31} \mathrm{~B}_{1}\right] & =\operatorname{span}\left[\mathrm{H}_{32} \mathrm{~B}_{2}\right]
\end{aligned}
$$

In the above formula, space composed of pre-coding and column vector of channel matrix is represented by the span[w]. In order to achieve the constraint requirements of interference alignment, following formula must be satisfied more strictly:

$$
\begin{aligned}
\operatorname{span}\left[\mathrm{H}_{12} \mathrm{~B}_{2}\right] & =\operatorname{span}\left[\mathrm{H}_{13} \mathrm{~B}_{3}\right] \\
\mathrm{H}_{21} \mathrm{~B}_{1} & =\mathrm{H}_{23} \mathrm{~B}_{3} \\
\mathrm{H}_{31} \mathrm{~B}_{1} & =\mathrm{H}_{32} \mathrm{~B}_{2}
\end{aligned}
$$

Formula (3.3) and formula (3.1) have the same meaning, which is only for the convenience of calculation of pre-coding matrix:

$$
\begin{gathered}
\operatorname{span}\left(B_{1}\right)=\operatorname{span}\left(E_{1}\right) \\
B_{2}=\left(\mathrm{H}_{32}\right)^{-1} \mathrm{H}_{31} \mathrm{~B}_{1} \\
\mathrm{~B}_{3}=\left(\mathrm{H}_{23}\right)^{-1} \mathrm{H}_{21} \mathrm{~B}_{1}
\end{gathered}
$$

Actually, $E=\left(\mathrm{H}_{31}\right)^{-1} \mathrm{H}_{32}\left(\mathrm{H}_{12}\right)^{-1} \mathrm{H}_{13}\left(\mathrm{H}_{23}\right)^{-1} \mathrm{H}_{21}$. From the formula " $\operatorname{span}\left(\mathrm{B}_{1}\right)=\operatorname{span}\left(\mathrm{EB}_{1}\right)$ " we can see that $\mathrm{B}_{1}$ is a feature vector of $\mathrm{E}$, therefore:

$$
B_{1}=\left[e 1, e 2 \ldots e n_{t} / 2\right]
$$

Among them, e1, e2..e $n_{t} / 2$ is E's feature vectors which amounts of $n_{t} / 2$. In the same way, we can calculate $\mathrm{B}_{2}$ and $\mathrm{B}_{3}$ from the two equations behind.

From the above analysis, we can know that the advantage of $\mathrm{CJ}$ interference alignment is eliminating the inter cell interference very well, which helping the degree of transmitting freedom to achieve the maximum level. However, CJ interference alignment ignored the impact of complex environment of wireless channel for the cell signal transmission, especially the defect will be significantly larger in the multiuser and multipoint cooperative transmission system. So, in this article, MSV-TZ pre-coding scheme is proposed under the premise of eliminating the interference of other cells of the residential customers. The scheme of the precoding matrix is singled out from the pre-coding vector collection which the residential user channel set in the best performance of a group of pre-coding vector.

In contrast to the conventional approach, the MSV-TZ scheme does not simply choose $n_{t} / 2$ feature vector for calculating pre-coding matrix, but using the singular vectors $\mathrm{H}_{\mathrm{ii}}$, which corresponding the largest $n_{t} / 2$ singular value, and choosing the feature vector of $E$, to calculate $\mathrm{B}_{1}$.

Using SVD to break down $\mathrm{H}_{\mathrm{ii}}$, we can get:

$$
H_{i i}=U A V^{H}=\left[U_{i 1} \cdots U_{i M}\right]\left[\begin{array}{lll}
a_{i 1} & & \\
& \ddots & \\
& & a_{i M}
\end{array}\right]\left[\begin{array}{c}
V_{i 1} \\
\vdots \\
V_{i M}
\end{array}\right]
$$

$\mathrm{A}$ is a singular value matrix, which the singular value is $a_{i m}, i=1,2,3, \mathrm{~m}=1, \ldots, n_{t}$ besides, $\mathrm{U}_{\mathrm{im}}$ and $\mathrm{V}_{\mathrm{im}}$ are the corresponding Left and right singular vectors of $\mathrm{U}$ and $\mathrm{V}$, where $\mathrm{i}$ is cell index, its range is from 1 to $3, \mathrm{~m}$ is the singular value, its range is from 1 to $\mathrm{n}_{\mathrm{t}} ; \mathrm{V}_{\mathrm{im}}$ represents the best qualities of $n_{t} / 2$ Eigen channels in cell; 
On the basis of formula (2.5), in order to transmit signals in the $n_{t} / 2$ sub channel which channel quality feature are best, we need to choose $n_{t} / 2$ feature vectors which have minimum chordal distance with $V_{\text {im }}$ to replace $V_{\text {im }}$ that:

$$
B_{1}=\underset{B_{1}<e i g(E)}{\operatorname{argmax}}\left\{\sum_{m=1}^{n_{t / 2}} a_{i m}\left\|\left(V_{i m}\right)^{H} B_{i m}\right\|\right\}
$$

The reason why we choose aim as weighting factor is that to eliminate the fact of weight from different feature channel [9]. In spite of $\mathrm{B}_{2}$ and $\mathrm{B}_{3}$ can be obtained according to equation (3.1) of the first 2 and 3 equation, it is not imperfect to get $B_{i}$ respectively, so this pre-coding matrix can only ensure the cell users without interference, and cannot exclude the influence of other cell sites and users. Therefore, the basis of choosing pre-coding in CoMP-MU-MIMO system should be that can enhance the performance of all the users in cells of the edge, as follows:

$$
\left\langle B_{1}, B_{2}, B_{3}\right\rangle=\underset{\substack{B_{1}<e i g(E) \\ B_{2}=\left(H_{32}\right)^{-1} H_{31} B_{1} \\ B_{3}=\left(H_{23}\right)^{-1} H_{21} B_{1}}}{\operatorname{argmax}}\left\{\sum_{i=1}^{3} \sum_{m=1}^{n_{t / 2}} a_{i m}\left\|\left(V_{i m}\right)^{H} B_{i m}\right\|\right\}
$$

Execution steps of MSV-TZ pre-coding algorithm are following:

1: SVD decompose channel matrix of each base station to the cell users;

2: Calculate pre-coding matrix of MSV-TZ scheme;

\section{Simulation and Analysis}

We use a system-level simulation to evaluation the performance of CoMP-MU-MIMO system, and assuming that use the same power to transmit all subcarrier. For detailed simulation parameters, refer to Table 1 [10]. In the simulation system, each user terminal has 2 receiving antennas, and each base node has 2 sending antennas, the number of CoMP-MU-MIMO is always 3 , thus 3 cooperative transmission nodes and three user terminals can form virtual CoMP-MU-MIMO system for $6 \times 6$. 


\begin{tabular}{|l|l|}
\hline Parameters & Value \\
\hline Layout & 3 -sectorized hexagonal grid with 7 sites \\
\hline Inter-side distance & $500 \mathrm{~m}$ \\
\hline Carrier frequency & $2.0 \mathrm{GHz}$ \\
\hline Bandwidth & $10 \mathrm{MHz}$ \\
\hline DL/UL subcarrier ration & $2 \mathrm{DL} / 2 \mathrm{UL}$ \\
\hline Ave.Num of users & 10 \\
\hline Num of node transmitting antenna & 2 \\
\hline Num of use receiving antenna & 2 \\
\hline Layer num of each use & Rank=2,no rank adaption \\
\hline Antenna pattern & Dual polarized antenna \\
\hline UE speed & $3 \mathrm{Km} / \mathrm{h}$ \\
\hline Penetration loss & $20 \mathrm{~dB}$ \\
\hline Frequency reuse & 1 \\
\hline Path loss & $128.1+37.61 \mathrm{~g}(\mathrm{~d})$, Min $70 \mathrm{~d}$ \\
\hline Dispatch Algorithm & Distributed data stream \\
\hline HARQ & Handing 7 users at the same time , Max 3 \\
& transmissions. \\
\hline Channel model & SCM-E \\
\hline
\end{tabular}

Table 1: Simulation Parameters

Compare to the traditional pre-coding algorithm ZF and $\mathrm{BD}$, this article proposed multiuser MSV-TZ pre-coding algorithm has an advantage on the same signal interference, shown as Figure 3.

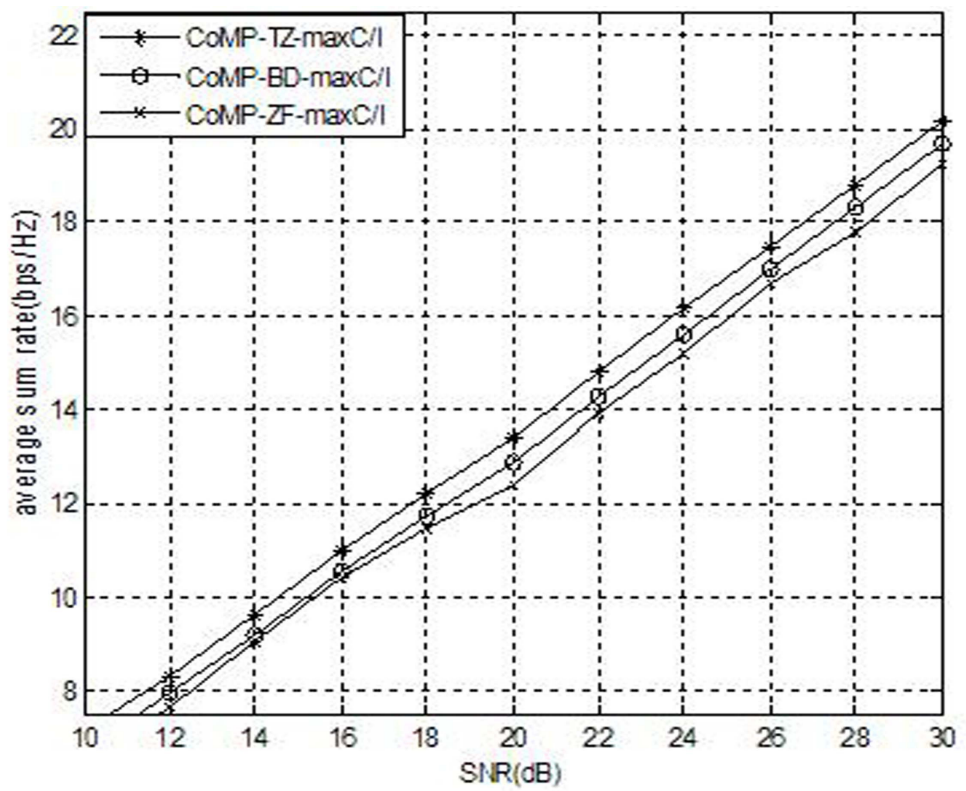

Figure 3: MSV-TZ Simulate Result

The scheme of the pre-coding matrix is singled out from the pre-coding vector collection which the residential user channel set in the best performance of a group of pre-coding vector.The signal to noise ratio in the measurements is very high, $20-30 \mathrm{~dB}$, due to the short transmission range [11]. The reason why the MSV-TZ scheme leads significant enhancement is that we are not simply choose $n_{t} / 2$ feature vector for calculating pre-coding matrix, but using the singular vectors $\mathrm{H}_{\mathrm{ii}}$ ( $\mathrm{i}$ is cell index), so more users will be served, and MSV-TZ precoding on the 
downlink can significantly increase the capacity of interference limited links by aligning all interfering signals at a given mobile in the same subspace which is orthogonal to the signal subspace.

MSV-TZ pre-coding scheme can be very good to eliminate interference from other cells, which can improve the spectral efficiency and system throughput of cell edge. Matlab simulation results can be seen that MSV-TZ pre-coding algorithm can significantly improve the throughput of the system, especially the edge user throughput.

\section{Conclusion}

We summarize the interference alignment issue on CoMP-MU-MIMO system which uses BBU+RRU model. Aiming at the deficiency of the classic interference alignment pre-coding algorithm, put forward a optimized plan based on the pre-coding matrix for characteristics of sub-channels, to improve the transmission signal strength. The simulation results show that, compared with the traditional pre-coding algorithm, this paper puts forward the optimization scheme makes the system throughput significantly improved.

\section{References}

[1] C. S. Vaze, M. K.Varanasi. The Degree-of-freedom Regions of MIMO Broadcast, Interference, and Cognitive Radio Channels with No CSIT [J]. IEEE Transactions on Information Theory. 58(8), 5354-5374(2012).

[2] Y. Zhu, D. N. Guo. The Degrees of Freedom of Isotropic MIMO Interference Channels Without State Information at the Transmitters [J]. IEEE Transactions on Information Theory. 58(2),341352(2012).

[3] R. Tandon, S. Mohajer, H. V. Poor, et al. Degrees of Freedom Region of the MIMO Interference Channel with Output Feedback and Delayed CSIT [J]. IEEE Transactions on Information Theory. 59(3),1444-1457(2013).

[4] Y. Zhang, Z. Zhou, L. Shi, et al. Interference alignment based on exact potential game[J]. Journal of Beijing University of Posts and Telecommunications. 36(2), 50-54(2013).

[5] B. Nazer, M. Gastpar, S. A. Jafar, et al. Ergodic Interference Alignment[J]. IEEE Transactions on Information Theor. 58(10),6355-6371(2012).

[6] X. Z. Xie, B. Xu, S. Ma. Three cell environment based on low-complexity algorithm for interference alignment triangular decomposition $[\mathrm{J}]$. Journal of Electronics \& Information Technology. 65(4),13-17(2013).

[7] S. M. Razavi, T. Ratnarajah. Performance analysis of interference alignment under CSI mismatch[J].IEEE Transactions on Vehicular Technology. 51(2), 535-537(2012).

[8] S. M. Razavi, T. Ratnarajah. Interference alignment in K-User MIMO interference channels with partially coordinated receivers[J]. IET Communications.8(1), 50-57(2014).

[9] X. Yi, S. Yang, D. Gesbert, M. Kobayashi. The degrees of freedom of temporally-correlated MIMO networks with delayed CSIT]J].IEEE Transactions on Information Theory. 60(1), 494514(2014).

[10]M. Mungara, G. George, A. Lozano. Overhead and spectral efficiency of pilot-assisted interference alignment in time-selective fading channels[J].IEEE Transactions on Wireless Communications. 52(2),311-312(2014). 
[11] M. Guillaud, M. Rezaee, G. Matz. Interference alignment via message-passing[C]. IEEE International Conference on Communications. Sydney Conference, Sydney. Pp,10-14,(2014). 